Pacific Journal of Mathematics

GENERALIZED RAMSEY THEORY FOR GRAPHS. III. SMALL
OFF-DIAGONAL NUMBERS 


\title{
GENERALIZED RAMSEY THEORY FOR GRAPHS, III. SMALL OFF-DIAGONAL NUMBERS
}

\author{
VÁClav ChVÁtal ANd Frank HaRARY
}

The classical Ramsey theory for graphs studies the Ramsey numbers $r(m, n)$. This is the smallest $p$ such that every 2coloring of the lines of the complete graph $K_{p}$ contains a green $K_{m}$ or a red $K_{n}$. In the preceding papers in this series, we developed the theory and calculation of the diagonal numbers $r(F)$ for a graph $F$ with no isolated points, as the smallest $p$ for which every 2-coloring of $K_{p}$ contains a monochromatic $F$. Here we introduce the off-diagonal numbers: $r\left(F_{1}, F_{2}\right)$ with $F_{1} \neq F_{2}$ is the minimum $p$ such that every 2coloring of $K_{p}$ contains a green $F_{1}$ or a red $F_{2}$. With the help of a general lower bound, the exact values of $r\left(F_{1}, F_{2}\right)$ are determined for all graphs $F_{i}$ with less than five points having no isolates.

1. Introduction. The small ( $p \leqq 4$ points) graphs $F_{i}$ having no isolated points are shown in Figure 1, together with their symbolic names, following the notation for operations on graphs in the book [3, p. 21]. In fact, we follow the terminology and notation of this book throughout.
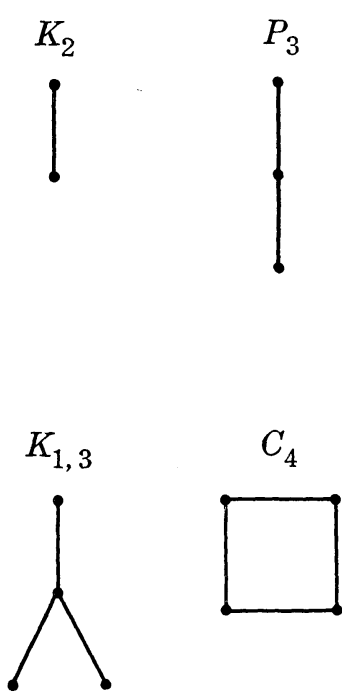

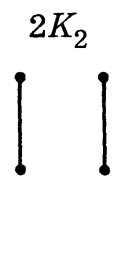

$K_{1,3}+x$

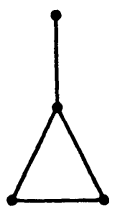

Figure 1

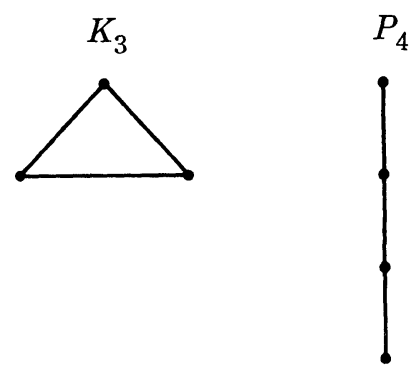

$K_{4}-x$

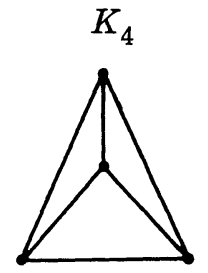

In $[1,2]$, we defined the number $r(F)$ as the minimum $p$ for which every 2-coloring (of the lines) of $K_{p}$ contains a monochromatic subgraph $F$. The number $r\left(F_{1}, F_{2}\right)$ is the corresponding smallest $p$ 
such that every 2-coloring of $K_{p}$ contains a green $F_{1}$ or a red $F_{2}$. Obviously $r(F)=r(F, F)$, so that the numbers $r(F)$ are diagonal within the $r\left(F_{1}, F_{2}\right)$.

There is an equivalent formulation of the definition of $r\left(F_{1}, F_{2}\right)$ in terms of graphical complementation rather than 2-colorings of a complete graph. Namely, $r\left(F_{1}, F_{2}\right)$ is the minimum $p$ such that whenever a $p$-point graph $G$ does not have $F_{1}$ as a subgraph, then its complement $\bar{G}$ contains $F_{2}$. It is convenient to assign numbers to the following immediate consequences of the definition: symmetry, monotonicity, and a crude lower bound,

$$
r\left(F_{1}, F_{2}\right)=r\left(F_{2}, F_{1}\right)
$$

$$
\begin{gathered}
F_{1}^{\prime} \subset F_{1} \text { and } F_{2}^{\prime} \subset F_{2} \text { imply } r\left(F_{1}^{\prime}, F_{2}^{\prime}\right) \leqq\left(F_{1}, F_{2}\right) \\
r\left(F_{1}, F_{2}\right) \geqq \max \left(p\left(F_{1}\right), p\left(F_{2}\right)\right) .
\end{gathered}
$$

When $F_{1}$ and $F_{2}$ are both complete graphs, we have specialized to $r\left(K_{m}, K_{n}\right)=r(m, n)$, the classical Ramsey numbers for graphs. As all the numbers $r(m, n)$ are known for $m, n=2,3,4$, we begin with some information about off-diagonal Ramsey numbers for small $F_{1}$ and $F_{2}$. The existence of the diagonal numbers $r(n, n)$ was established by Ramsey [4] himself; that of all the other numbers $r\left(F_{1}, F_{2}\right)$ follows from (2).

From [3, p. 17], we have the following values of $r(m, n)$ :

\begin{tabular}{r|rrr}
$n$ & 2 & 3 & 4 \\
\hline 2 & 2 & 3 & 4 \\
3 & & 6 & 9 \\
4 & & & 18
\end{tabular}.

In [2], the numbers $r(F)$ are determined for the 10 graphs of Fig. 1:

$$
\begin{array}{c|cccccccccc}
F & K_{2} & P_{3} & 2 K_{2} & K_{3} & P_{4} & K_{1,3} & C_{4} & K_{1,3}+x & K_{4}-x & K_{4} \\
r(F) & 2 & 3 & 5 & 6 & 5 & 6 & 6 & 7 & 10 & 18 .
\end{array}
$$

It is obvious that $r\left(K_{2}, F\right)=p(F)$, the number of points in $F$.

2. The simplest Ramsey numbers. We now obtain two equations which give the next two rows in Table 1.1, the first for Ramsey numbers involving $2 K_{2}$ and the second for $P_{3}$.

Lemma 1. For any graph $F$ with no isolates, 


$$
r\left(2 K_{2}, F\right)= \begin{cases}p(F)+2 & \text { if } F \text { is complete } \\ p(F)+1 & \text { otherwise. }\end{cases}
$$

Proof. First, when $F$ is complete, we have $r\left(2 K_{2}, F\right)>p(F)+1$ because a 2-coloring of $K_{p+1}$ in which the green lines form just one triangle cannot have a red $K_{p}$. On the other hand, if a 2-coloring of $K_{p+2}$ has no green $2 K_{2}$, then the green lines form either a star or a triangle, so there must be a red $K_{p}$.

Secondly when $F$ is not complete, it is a subgraph of $K_{p}-x$. In an arbitrary 2-coloring of $K_{p+1}$ which does not contain a green $2 K_{2}$, the green lines again form a star or a triangle. When there is a green star, there must be a red $K_{p}$. And when we have a green triangle, there must appear a green $K_{p}-x$. Thus $r\left(2 K_{2}, F\right) \leqq p(F)+1$. The equality follows from the 2-coloring of $K_{p}$ with red $K_{p-1}$ and a green star $K_{1, p-1}$.

The next question is a bit more subtle.

Lemma 2. For any graph $F$ with no isolates,

$$
r\left(P_{3}, F\right)=\left\{\begin{array}{l}
p(F) \text { if } F \text { has a 1-factor } \\
2 p(F)-2 \beta_{1}(F)-1 \text { otherwise. }
\end{array}\right.
$$

Proof. In each 2-coloring of $K_{m}$ without a green $P_{3}$, all the green lines are independent. In other words, the green graph is a subgraph of $[m / 2] K_{2}$ or, equivalently, the red graph contains $K_{m}-[m / 2] K_{2}$. (For $m$ even, this graph has been called a "party graph" by A. J. Hoffman because everyone talks to everyone else with the exception that nobody talks to his own spouse.) Thus, $r\left(P_{3}, F\right)$ is the smallest $m$ such that $F$ is a subgraph of $K_{m}-[m / 2] K_{2}$.

For any graph $F$ with $p$ points, we have the maximum number of independent lines in the complement of $F, \beta_{1}(\bar{F})=n$ if and only if $F \subset K_{p}-n K_{2}$. Thus, if $\bar{F}$ has a 1 -factor, i.e., $\beta_{1}(\bar{F})=p / 2$, then we have $F \subset K_{p}-(p / 2) K_{2}$ or $r\left(P_{3}, F\right) \leqq p$. The equality follows trivially from (2).

Now, let $\bar{F}$ have no 1 -factor, so that $\beta_{1}(\bar{F})=n<p / 2$. If $m=$ $2 p-2 n-1$, then any 2-coloring of $K_{m}$ having no green $P_{3}$ has a red $K_{m}-[m / 2] K_{2}=K_{m}-(p-n-1) K_{2}$. We will show that such a coloring has a red $F$. Starting with the simple inclusion $(p-n-1) K_{2} \cup K_{1} \subset$ $n K_{2} \cup(p-2 n) K_{1}$, and taking complements by merely removing the indicated number of independent lines from a complete graph of the proper size, we obtain $K_{p}-n K_{2} \subset K_{m}-(p-n-1) K_{2}$. Thus, we have $r\left(P_{3}, F\right) \leqq 2 p-2 n-1$. On the other hand, the 2-coloring of $K_{m-1}$ which has just $(m-1) / 2=p-n-1$ green independent lines 
and leaves as the remaining red graph $K_{m-1}-((m-1) / 2) K_{2}$ already has no green $P_{3}$. It contains no red $F$ either, for otherwise $((m-1) / 2) K_{2} \subset$ $\bar{F}$ or equivalently $n=\beta_{1}(\bar{F})>(m-1) / 2=p-n-1$, contradicting $n<p / 2$ and proving Lemma 2 .

3. A useful lower bound. For our last lemma, we easily derive a simple lower bound which is not at all sharp in general, but luckily happens to be rather useful in establishing the values of $r\left(F_{1}, F_{2}\right)$ for the 10 small graphs of Fig. 1.

LEMMA 4. Let $F_{1}$ and $F_{2}$ be two graphs (not necessarily different) with no isolated points. Let $c$ be the number of points in a largest connected component of $F_{1}$, and let $\chi$ be the chromatic number of $F_{2}$. Then the following lower bound holds:

$$
r\left(F_{1}, F_{2}\right) \geqq(c-1)(\chi-1)+1 .
$$

Proof. Consider the graph $G=(\chi-1) K_{c-1}$. Since $G$ has no component with at least $c$ points, it cannot possibly contain $F_{1}$. On the other hand, the complement $\bar{G}$ is $(\chi-1)$-chromatic and hence cannot contain the $\chi$-chromatic graph $F_{2}$. The inequality follows at once, as $G$ has $(c-1)(\chi-1)$ points.

Remarkably, we shall find that in all but the two instances $r\left(K_{13}, C_{4}\right) \geqq 4$ and $r\left(K_{4}-x, K_{4}\right) \geqq 10$, this lower bound turns out to yield the exact number for $r\left(F_{1}, F_{2}\right)$.

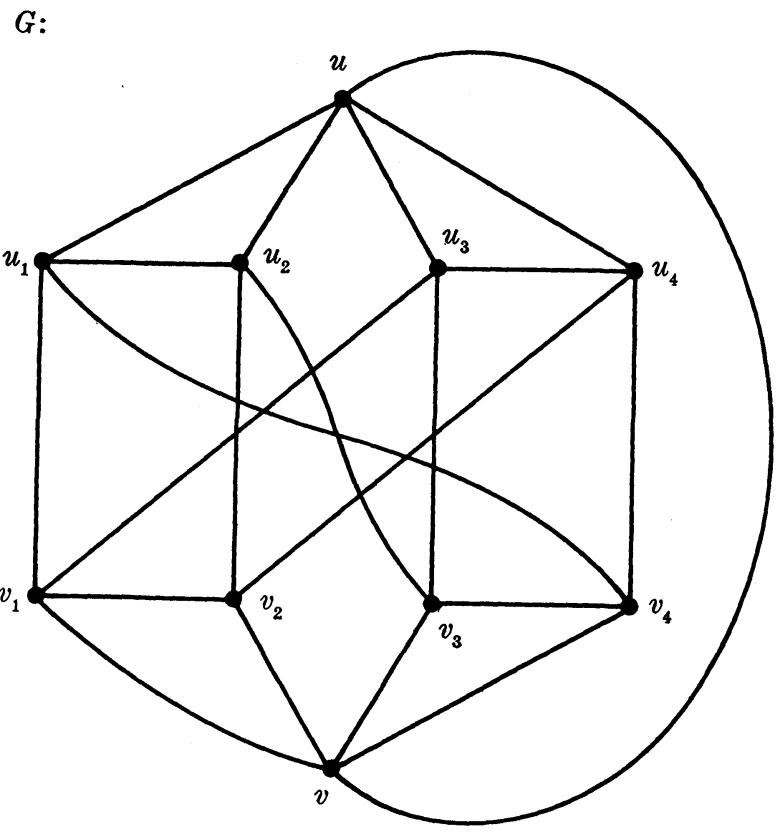

FIGURE 2 
Referring to Table 2 below, we next show that better lower bounds than 4 and 10 respectively are given by

$$
\begin{gathered}
r\left(K_{1,3}, C_{4}\right) \geqq 6 \\
r\left(K_{4}-x, K_{4}\right) \geqq 11 .
\end{gathered}
$$

Later we will see that (4) and (5) give the correct values of these two Ramsey numbers.

To prove (4) we need only exhibit a graph $G$ with 5 points such that $G$ has no $K_{1,3}$ (i.e., no point of degree exceeding 2) and $\bar{G}$ has no 4-cycle. Clearly $G=C_{5}$ works.

Similarly (5) can be verified by producing $G$ with 10 points not containing $K_{4}-x$ such that $\beta_{0}(G)<4$. This example is a bit trickier, but we finally found it.

The graph $G$ of Fig. 2 has just four triangles, no two having a common line. Hence $G$ does not contain $K_{4}-x$. It is also easily seen that $G$ has no set of 4 independent points.

4. Forcing forbidden subgraphs. For each pair $F_{1}, F_{2}$ of forbidden graphs, we must argue that when the number $r$ of points is right, every graph $G$ with $r$ points not containing $F_{1}$ must have $F_{2}$ in its complement. In particular, we will prove the next 8 upper bounds which establish the remaining off-diagonal Ramsey numbers.

$$
\begin{gathered}
r\left(P_{4}, K_{1,3}\right) \leqq 5 \\
r\left(P_{4}, C_{4}\right) \leqq 5 \\
r\left(K_{1,3}, C_{4}\right) \leqq 6 \\
r\left(K_{1,3}+x, K_{4}-x\right) \leqq 7 \\
r\left(C_{4}, K_{4}-x\right) \leqq 7 \\
r\left(K_{1,3}+x, K_{4}\right) \leqq 10 \\
r\left(C_{4}, K_{4}\right) \leqq 10 \\
r\left(K_{4}-x, K_{4}\right) \leqq 11 .
\end{gathered}
$$

Proof of (6) and (7). By coincidence, both (6) and (7) may be shown at one fell swoop. Let $G$ have no 4 -point path $P_{4}$ on its 5 points. There are only two possibilities for such a graph: either $G \subset$ $K_{2} \cup K_{3}$ or $G \subset K_{14}$. Taking complements, $K_{2,3} \subset \bar{G}$ or $K_{4} \subset \bar{G}$, so that necessarily both $K_{1,3}$ and $C_{4}$ are subgraphs of $\bar{G}$.

Proof of (8). Taking $G$ as a 6 -point graph with all degrees $\leqq 2$ 
forces $\bar{G}$ to have each degree $\geqq 3$. Thus, in $\bar{G}$, the neighborhoods of any two nonadjacent points have at least two common points, so that $\bar{G}$ must contain $C_{4}$.

The next assertion (9) will automatically have several consequences by the monotonicity condition (2).

Proof of (9). Let $G$ be an arbitrary graph of 7 points not containing $K_{1.3}+x$. We assume $\bar{G}$ does not contain $K_{4}-x$ and proceed to derive a contradiction. There are two possibilities, depending on whether $G \supset K_{3}$. If $G$ does have a triangle $u_{1} u_{2} u_{3}$, with the remaining points labeled $v_{j}$, then there can be no line $u_{i} v_{j}$ in $G$. Now each pair of the points $v_{j}$ is forced to be adjacent in $G$, for otherwise $\bar{G}$ would contain $K_{4}-x$. Hence the points $v_{j}$ induce $K_{4}$ in $G$, a contradiction.

Next, if $G$ has no triangle, then it has 3 independent points $u_{1}$, $u_{2}, u_{3}$ since $r\left(K_{3}, K_{3}\right)=r\left(K_{3}\right)=6$. Again, we denote the remaining four points by $v_{j}$. Each $v_{j}$ must be adjacent in $G$ to at least two of the points $u_{i}$, for otherwise $G \supset K_{4}-x$. If there is even one line $v_{i} v_{j}$, then $G$ contains $K_{1,3}+x$, contrary to the hypothesis. Thus $\bar{G}$ is forced to contain $K_{4}$, and a fortiori $K_{4}-x$.

We now apply (2) and the inclusions

$$
K_{13}+x \supset K_{13}, P_{4}, K_{3}
$$

to (9) to obtain at once the lower bounds

$$
\begin{array}{r}
r\left(K_{3}, K_{4}-x\right) \leqq 7 \\
r\left(P_{4}, K_{4}-x\right) \leqq 7 \\
r\left(K_{1,3}, K_{4}-x\right) \leqq 7 .
\end{array}
$$

Similarly $K_{4}-x \supset K_{1,3}+x, C_{4}, K_{1,3}, P_{4}$ and (2) applied to (14) give

$$
\begin{gathered}
r\left(K_{3}, P_{4}\right) \leqq 7 \\
r\left(K_{3}, K_{1,3}\right) \leqq 7 \\
r\left(K_{3}, C_{4}\right) \leqq 7 \\
r\left(K_{3}, K_{1,3}+x\right) \leqq 7 .
\end{gathered}
$$

Similarly by (15),

$$
r\left(P_{4}, K_{1,3}+x\right) \leqq 7,
$$

and by (16), 


$$
r\left(K_{1,3}, K_{1,3}+x\right) \leqq 7
$$

Proof of (10). Let $G$ be an arbitrary graph with 7 points and no $C_{4}$. We will assume $\bar{G} \not \supset K_{4}-x$ and deduce a contradiction.

In the proof, we distinguish two cases according to whether there is or is not a point $u$ of degree smaller than three. In the first case, we delete the point $u$ together with its neighbors and are left with a subgraph $H$ of $G$ having at least four points. Clearly, $H$ has no $C_{4}$ because $G$ has none. Thus, as $r\left(P_{3}, C_{4}\right)=4$ by Lemma $2, \bar{H}$ is forced to contain $P_{3}$. By definition of $H, u$ is adjacent to no point in $H$. Therefore, $\bar{G}$ contains $K_{4}-x$, contradicting the assumption.

Next, we consider the second case where each point in $G$ has degree at least three. Now the inequality (9), $r\left(K_{1_{3}}+x, K_{4}-x\right) \leqq 7$, proved above, implies $K_{1,3}+x \subset G$. A fortiori, $G$ contains a triangle $u_{1} u_{2} u_{3}$. Now, since each point of $G$ has degree at least three and $G$ contains no $C_{4}$, we conclude that there are three other points $v_{1}, v_{2}, v_{3}$ such that $u_{i} v_{i}$ is a line of $G$ for each $i=1,2,3$. In other words, $G$ contains the subgraph shown in Figure 3. Actually, it is easy to check that the graph in Fig. 3 is the subgraph of $G$ induced by $u_{1}, u_{2}, u_{3}$, $v_{1}, v_{2}, v_{3}$, for the addition of any line to this graph produces $C_{4}$. But then $\bar{G}$ contains $K_{4}-\imath$ with points $u_{1}, v_{1}, v_{2}, v_{3}$ again contradicting the assumption.

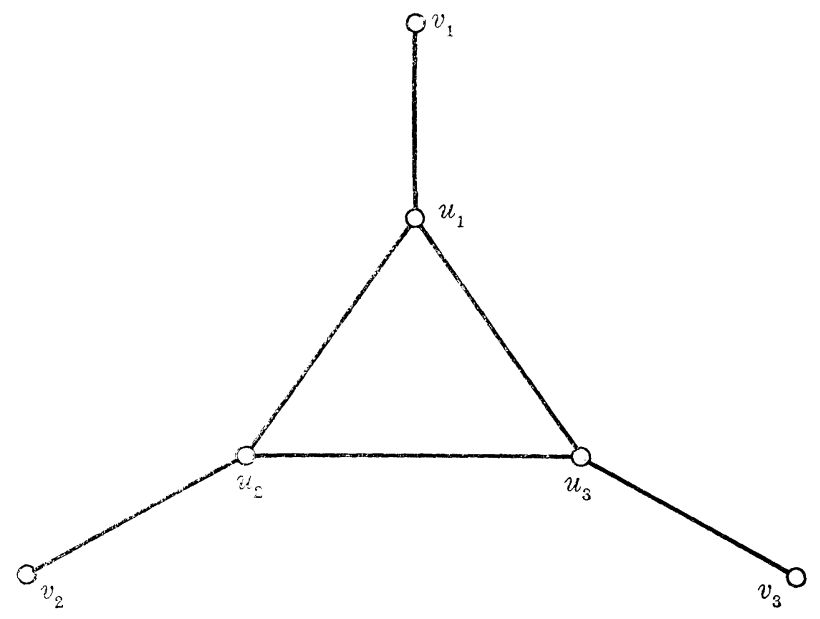

Figure 3

Proof of (11). Assume there is a graph $G$ with 10 points such that $G$ contains no $K_{1_{3}}+\imath$ and $\beta_{0}(G)<4$. As $r\left(K_{s}, K_{4}\right)=\imath(3,4)=9, G$ contains a triangle $u_{1} u_{2} u_{3}$. Let the other points in $G$ be $v_{j}(j=1,2$,

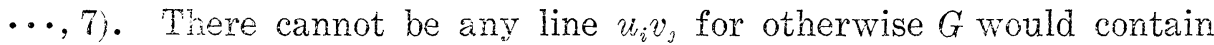
a $K_{13}+\cdots$. Now, let us consider the subgraph $H$ of $G$ spanned by the 
$v_{j}$ 's. $H$ has 7 points and no $K_{1,3}+x$ because $G$ has none. Thus, the inequality (20) written in the form $r\left(K_{1,3}+x, K_{3}\right) \leqq 7$ implies the existence of three independent $v_{j}$ 's. Since $u_{1}$ is adjacent to none of these, we then have $\beta_{0}(G) \geqq 4$, contrary to the initial assumptions, completing the proof of (11).

Now we can apply (2) and the inclusions $K_{1,3}+x \supset K_{1,3}, P_{4}$ to (11) to obtain two more upper bounds,

$$
\begin{aligned}
& r\left(K_{1,3}, K_{4}\right) \leqq 10 \\
& R\left(P_{4}, K_{4}\right) \leqq 10 .
\end{aligned}
$$

It is quite convenient to have another lemma for the proof of (12).

LEMma 3. If a graph $G$ with $p$ points has minimum degree $d$ and $d(d-1)>p-1$, then $G$ contains $C_{4}$.

Proof. Let $n$ be the total number of paths $P_{3}$ contained in $G$. There are exactly $p$ choices for the midpoint of $P_{3}$, and for each fixed midpoint at least $\left(\begin{array}{l}d \\ 2\end{array}\right)$ choices of the endpoints. Therefore $n \geqq p\left(\begin{array}{l}d \\ 2\end{array}\right)>$ $\left(\begin{array}{l}p \\ 2\end{array}\right)$ so there must be two distinct paths $P_{3}$ in $G$ with the same pair of endpoints, and hence a cycle $C_{4}$.

Proof of (12). Let $G$ be a graph with 10 points such that the point independence number $\beta_{0}(G)<4$. Then necessarily the chromatic number $\chi(G) \geqq 4$. Hence by Brooks' Theorem, see [3, p. 128], either $K_{4}$ (and hence $C_{4}$ ) is contained in $G$, or the degree of each point of $G$ is at least four in which case the conclusion follows from Lemma 3.

Proof of (13). We have to show that there is no graph $G$ with 11 points such that $K_{4}-x \not \subset G$ and $\beta_{0}(G)<4$, so again we assume the contrary. Our first aim is to show that $G$ must be regular of degree 4 . This will be done by degrees, considered as possible separate cases.

Case 1. G has a point $u$ of degree $\geqq 7$. Then the neighborhood subgraph $H$ of $u$ (induced by the neighborhood of $u$ ) has at least 7 points and clearly contains no set of four independent points. By Lemma $2, r\left(P_{3}, K_{4}\right)=7$, so $H$ must contain $P_{3}$, which on joining $u$ implies $K_{4}-x \subset G$. This contradiction proves the impossibility of Case 1.

Case 2. $G$ has a point $u$ of degree 6 . Then the neighborhood 
subgraph $H$ of $u$ has exactly six points, no four of them being independent. As $G$ contains no $K_{4}-x, H$ cannot contain $P_{3}$. It is easy to see that these conditions imply $H=3 K_{2}$; let the three independent lines of $H$ be $v_{1} w_{1}, v_{2} w_{2}$ and $v_{3} w_{3}$. There are four other points in $G$; call one of them $u_{0}$. This point cannot be adjacent to both $v_{i}$ and $w_{i}$ for some $i \in\{1,2,3\}$ since otherwise $G$ would contain $K_{4}-x$. Thus, we may assume $u_{0}$ not adjacent to $v_{1}, v_{2}, v_{3}$. But then the points $u_{0}, v_{1}, v_{2}, v_{3}$ are independent contradicting $\beta_{0}(G)<4$. Hence the asumption of Case 2 is false.

Case 3. $G$ has a point $u$ of degree 5. Similarly as above, we can prove that the neighborhood graph $H$ of $u$ must be $2 K_{2} \cup K_{1}$. Let its two lines be $u_{1} v_{1}$ and $u_{2} v_{2}$, and let its fifth point be $w$. There are five other points in $G$. If all of them are adjacent to $w$, then the degree of $w$ equals six. As we saw, this assumption led to a contradiction in Case 2. Thus there is a point $w_{0}$ adjacent neither to $u$ nor to $v$. Clearly, $w_{0}$ cannot be adjacent to both $u_{1}$ and $v_{1}$ (nor to both $u_{2}$ and $v_{2}$ ) as otherwise $G$ would contain $K_{4}-x$. Thus, we may assume $w_{0}$ not adjacent to $u_{1}, u_{2}$. But then $w_{0}, w, u_{1}$ and $u_{2}$ form a set of four independent points, contradicting $\beta_{0}(G)<4$.

Finally, to rule out any degree other than 4 , we consider

Case 4. $G$ contains a point $u$ of degree $\leqq 3$. Then there is a set $S$ of seven points in $G$ which are distinct from $u$ and not adjacent to $u$. The subgraph $\langle S\rangle$ of $G$ induced by $S$ contains no $K_{4}-x$. Since by (14), $r\left(K_{4}-x, K_{3}\right) \leqq 7,\langle S\rangle$ necessarily contains three independent points $u_{1}, u_{2}, u_{3}$ and hence $G$ contains four independent points, namely $u, u_{1}, u_{2}, u_{3}$ contradicting $\beta_{0}(G)<4$.

We have shown that each of the Cases 1-4 leads to a contradiction. Therefore, $G$ must be regular of degree 4 . Clearly, every line of $G$ is contained in at most one triangle, for otherwise $G$ would contain $K_{4}-x$. On the other hand, if every line of $G$ is in exactly one triangle, then the number of lines of $G$ would be divisible by three. However, $G$ has 22 edges and so it has a line, say $u v$, contained in no triangle. Let the other three neighbors of $u$ be $u_{1}, u_{2}, u_{3}$ and let the other three neighbors of $v$ be $v_{1}, v_{2}, v_{3}$. As $u v$ is contained in no triangle, all these are distinct. Now, we show that the subgraph of $G$ spanned by $u_{1}, u_{2}, u_{3}$ must contain exactly one line. For if it has none, then the points $u_{1}, u_{2}, u_{3}, v$ would be independent; if it has more than one, then $G$ would contain $K_{4}-x$ with points $u, u_{1}, u_{2}, u_{3}$. Similarly, the subgraph of $G$ spanned by $v_{1}, v_{2}, v_{3}$ also contains exactly one line. Let these two lines be $u_{1} u_{2}$ and $v_{1} v_{2}$. Next, let $w$ be one of the remaining three points $w_{1}, w_{2}, w_{3}$ in $G$. This point cannot be adjacent to both $u_{1}$ and $u_{2}$ for $G$ would then contain $K_{4}-x$. 
Thus, we may assume $w$ not adjacent to $u_{1}$. If $w$ is not adjacent to $u_{3}$, then $u_{1}, u_{3}, w, v$ are four independent points, contradicting $\beta_{0}(G)<4$. So $w$ must be adjacent to $u_{3}$. As $w$ is arbitrary, we conclude that each of the points $w_{1}, w_{2}, w_{3}$ is adjacent to $u_{3}$. By a symmetry argument, each of $w_{1}, w_{2}, w_{3}$ is adjacent to $v_{3}$. Then there can be no line $w_{i} w_{j}$ in $G$, for otherwise $F$ would contain $K_{4}-x$ with points $u_{3}, v_{3}, w_{i}, w_{j}$. Thus the points $w_{1}, w_{2}, w_{3}$ are independent. But then the points $u, w_{1}, w_{2}, w_{3}$ are independent, contradicting $\beta_{0}<4$.

5. Conclusions. The following table summarizes the results obtained (for both diagonal and off-diagonal) generalized Ramsey numbers.

TABLE 2. Small generalized Ramsey numbers

\begin{tabular}{l|rrrrrrrrrr}
\hline & $K_{2}$ & $P_{3}$ & $2 K_{2}$ & $K_{3}$ & $P_{4}$ & $K_{1,3}$ & $C_{4}$ & $K_{1,3}+x$ & $K_{4}-x$ & $K_{4}$ \\
\hline$K_{2}$ & 2 & 3 & 4 & 3 & 4 & 4 & 4 & 4 & 4 & 4 \\
$P_{3}$ & & 3 & 4 & 5 & 4 & 5 & 4 & 5 & 5 & 7 \\
$2 K_{2}$ & & & 5 & 5 & 5 & 5 & 5 & 5 & 5 & 6 \\
$K_{3}$ & & & & 6 & 7 & 7 & 7 & 7 & 7 & 9 \\
$P_{4}$ & & & & & 5 & 5 & 5 & 7 & 7 & 10 \\
$K_{1,3}$ & & & & & & 6 & 6 & 7 & 7 & 10 \\
$C_{4}$ & & & & & & & 6 & 7 & 7 & 10 \\
$K_{1,3}+x$ & & & & & & & & 7 & 7 & 10 \\
$K_{4}-x$ & & & & & & & & & 10 & 11 \\
$K_{4}$ & & & & & & & & & & 18 \\
\hline
\end{tabular}

Notice the irregularity of the behavior of $r\left(F_{1}, F_{2}\right)$ :

$$
r\left(P_{4}, K_{3}\right)>r\left(P_{4}, P_{4}\right), r\left(K_{3}, K_{3}\right) \text {. }
$$

On the other hand,

$$
r\left(P_{3}, P_{3}\right)<r\left(P_{3}, K_{3}\right)<r\left(K_{3}, K_{3}\right)
$$

(inequalities which continue to hold when all subscripts are increased to 4). These suggest the following

Conjecture. For any graphs $F_{1}, F_{2}$ with no isolates,

$$
r\left(F_{1}, F_{2}\right) \geqq \min \left(r\left(F_{1}\right), r\left(F_{2}\right)\right) .
$$

It would be a formidable task indeed to extend this table to all 23 of the 5-point graphs with no isolates. In particular this would include the determination (exact, of course) of $r(5,5)$ which appears not intractable, but extremely complicated. Our experience show that some of these 5-point graphs will be more delicate to handle than 
others. Unless and until some more analytic, powerful, and automatic method is found for calculating the numbers $r\left(F_{1}, F_{2}\right)$, it is highly unlikely that these will be found for all the 6-point graphs and larger ones.

\section{REFERENCES}

1. V. Chvátal and F. Harary, Generalized Ramsey theory for graphs, I, Diagonal numbers. Periodica Math. Hungar., to appear.

2. - Generalized Ramsey theory for graphs, II, Small diagonal numbers, Proc. Amer. Math. Soc., 32 (1972), 389-394.

3. F. Harary, Graph Theory, Addison-Wesley, Reading, Mass, 1969.

4. F. P. Ramsey, On a problem of formal logic, Proc. London Math. Soc., 30 (1930), 264-286.

Received February 23, 1971. Research of the second author was supported in part by Grant AF 68-1515 from the Air Force Office of Scientific Research.

UNIVERSITY OF WATERLOO

AND

UNIVERSITY OF MICHIGAN 



\section{PACIFIC JOURNAL OF MATHEMATICS}

\section{EDITORS}

\author{
H. SAMELSON \\ Stanford University \\ Stanford, California 94305

\section{R. HoвBY} \\ University of Washington \\ Seattle, Washington 98105
}

\section{J. DugundJI}

Department of Mathematics University of Southern California Los Angeles, California 90007

\author{
RichaRd ARENS \\ University of California \\ Los Angeles, California 90024
}

\section{ASSOCIATE EDITORS}
E. F. BECKENBACH
B. H. NEUMANN
F. WOLF
K. YoshidA

\section{SUPPORTING INSTITUTIONS}

\author{
UNIVERSITY OF BRITISH COLUMBIA \\ CALIFORNIA INSTITUTE OF TECHNOLOGY \\ UNIVERSITY OF CALIFORNIA \\ MONTANA STATE UNIVERSITY \\ UNIVERSITY OF NEVADA \\ NEW MEXICO STATE UNIVERSITY \\ OREGON STATE UNIVERSITY \\ UNIVERSITY OF OREGON \\ OSAKA UNIVERSITY
}

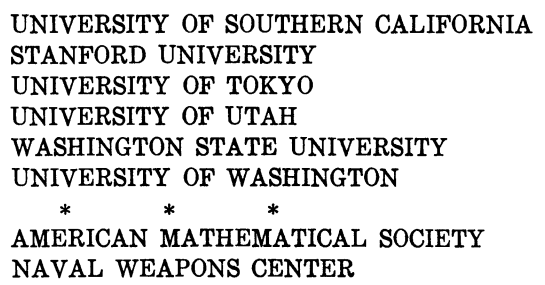

The Supporting Institutions listed above contribute to the cost of publication of this Journal, but they are not owners or publishers and have no responsibility for its content or policies.

Mathematical papers intended for publication in the Pacific Journal of Mathematics should be in typed form or offset-reproduced, (not dittoed), double spaced with large margins. Underline Greek letters in red, German in green, and script in blue. The first paragraph or two must be capable of being used separately as a synopsis of the entire paper. The editorial "we" must not be used in the synopsis, and items of the bibliography should not be cited there unless absolutely necessary, in which case they must be identified by author and Journal, rather than by item number. Manuscripts, in dup icate if possible, may be sent to any one of the four editors. Please classify according to the scheme of Math. Rev. Index to Vol. 39. All other communications to the editors should be addressed to the managing editor, Richard Arens, University of California, Los Angeles, California, 90024.

50 reprints are provided free for each article; additional copies may be obtained at cost in multiples of 50 .

The Pacific Journal of Mathematics is published monthly. Effective with Volume 16 the price per volume (3 numbers) is $\$ 8.00$; single issues, $\$ 3.00$. Special price for current issues to individual faculty members of supporting institutions and to individual members of the American Mathematical Society: $\$ 4.00$ per volume; single issues $\$ 1.50$. Back numbers are available.

Subscriptions, orders for back numbers, and changes of address should be sent to Pacific Journal of Mathematics, 103 Highland Boulevard, Berkeley, California, 94708.

PUBLISHED BY PACIFIC JOURNAL OF MATHEMATICS, A NON-PROFIT CORPORATION

Printed at Kokusai Bunken Insatsusha (International Academic Printing Co., Ltd.), 270, 3-chome Totsuka-cho, Shinjuku-ku, Tokyo 160, Japan. 


\section{Pacific Journal of Mathematics}

\section{Vol. 41, No. 2 December, 1972}

Tom M. (Mike) Apostol, Arithmetical properties of generalized Ramanujan sums .......................................... 281

David Lee Armacost and William Louis Armacost, On p-thetic groups ........ 295

Janet E. Mills, Regular semigroups which are extensions of groups .......... 303

Gregory Frank Bachelis, Homomorphisms of Banach algebras with minimal ideals ................................................ 307

John Allen Beachy, A generalization of injectivity .................. 313

David Geoffrey Cantor, On arithmetic properties of the Taylor series of rational functions. II.........................................

Václáv Chvátal and Frank Harary, Generalized Ramsey theory for graphs. III.

Small off-diagonal numbers .................................. 335

Frank Rimi DeMeyer, Irreducible characters and solvability of finite groups . . . . 347

Robert P. Dickinson, On right zero unions of commutative semigroups........ 355

John Dustin Donald, Non-openness and non-equidimensionality in algebraic

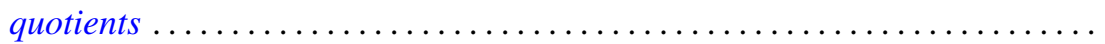

John D. Donaldson and Qazi Ibadur Rahman, Inequalities for polynomials with a prescribed zero ........................................ 375

Robert E. Hall, The translational hull of an $N$-semigroup ................ 379

John P. Holmes, Differentiable power-associative groupoids.............. 391

Steven Kenyon Ingram, Continuous dependence on parameters and boundary data for nonlinear two-point boundary value problems .

Robert Clarke James, Super-reflexive spaces with bases ..........

Gary Douglas Jones, The embedding of homeomorphisms of the plane in

continuous flows...............................

Mary Joel Jordan, Period $H$-semigroups and $t$-semisimple periodic

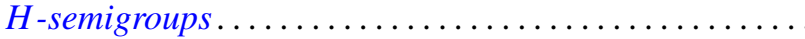

Ronald Allen Knight, Dynamical systems of characteristic 0

Kwangil Koh, On a representation of a strongly harmonic ring by sheaves...

Hui-Hsiung Kuo, Stochastic integrals in abstract Wiener space. ..

Thomas Graham McLaughlin, Supersimple sets and the problem of extending a

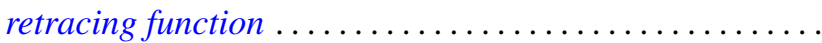

William Nathan, Open mappings on 2-manifolds .

M. J. O'Malley, Isomorphic power series rings

Sean B. O'Reilly, Completely adequate neighborhood systems and metrization

Qazi Ibadur Rahman, On the zeros of a polynomial and its derivative...

Russell Daniel Rupp, Jr., The Weierstrass excess function ..

Hugo Teufel, A note on second order differential inequalities and functional

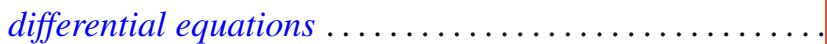

M. J. Wicks, A general solution of binary homogeneous equations over free 